\title{
Robust extraction of image correspondences exploiting the image scene geometry and approximate camera orientation
}

\author{
Bashar Alsadik $^{\mathrm{a}, \mathrm{c}}$, Fabio Remondino ${ }^{\mathrm{b}}$, Fabio Menna $^{\mathrm{b}}$, Markus Gerke ${ }^{\mathrm{a}}$, George Vosselman ${ }^{\mathrm{a}}$ \\ ${ }^{a}$ University of Twente, ITC Faculty, EOS department, Enschede, The Netherlands - (alsadik, gerke, vosselman)@itc.nl \\ ${ }^{\mathrm{b}}$ Bruno Kessler Foundation FBK, 3D Optical Metrology unit, Trento, Italy - (remondino,fmenna)@ fbk.eu \\ ${ }^{\mathrm{c}}$ University of Baghdad, College of Engineering, Surveying Department, Baghdad, Iraq
}

Commission V, WG V/4

KEY WORDS: IBM - Bundle adjustment - SIFT - 3D image - spanning tree

\begin{abstract}
:
Image-based modeling techniques are an important tool for producing 3D models in a practical and cost effective manner. Accurate image-based models can be created as long as one can retrieve precise image calibration and orientation information which is nowadays performed automatically in computer vision and photogrammetry. The first step for orientation is to have sufficient correspondences across the captured images. Keypoint descriptors like SIFT or SURF are a successful approach for finding these correspondences. The extraction of precise image correspondences is crucial for the subsequent image orientation and image matching steps. Indeed there are still many challenges especially with wide-baseline image configuration. After the extraction of a sufficient and reliable set of image correspondences, a bundle adjustment is used to retrieve the image orientation parameters.

In this paper, a brief description of our previous work on automatic camera network design is initially reported. This semi-automatic procedure results in wide-baseline high resolution images covering an object of interest, and including approximations of image orientations, a rough 3D object geometry and a matching matrix indicating for each image its matching mates. The main part of this paper will describe the subsequent image matching where the pre-knowledge on the image orientations and the pre-created rough $3 \mathrm{D}$ model of the study object is exploited. Ultimately the matching information retrieved during that step will be used for a precise bundle block adjustment.

Since we defined the initial image orientation in the design of the network, we can compute the matching matrix prior to image matching of high resolution images. For each image involved in several pairs that is defined in the matching matrix, we detect the corners or keypoints and then transform them into the matching images by using the designed orientation and initial 3D model. Moreover, a window is defined for each corner and its initial correspondence in the matching images. A SIFT or SURF matching is implemented between every matching window to find the homologous points. This is followed by Least Square Matching LSM to refine the correspondences for a sub-pixel localization and to avoid inaccurate matches. Image matching is followed by a bundle adjustment to orient the images automatically to finally have a sparse 3D model. We used the commercial software Photomodeler Scanner 2010 for implementing the bundle adjustment since it reports a number of accuracy indices which are necessary for the evaluation purposes. The experimental test of comparing the automated image matching of four pre-designed streopairs shows that our approach can provide a high accuracy and effective orientation when compared to the results of commercial and open source software which does not exploit the pre-knowledge about the scene.
\end{abstract}

\section{INTRODUCTION}

Image-based modeling (IBM) (Remondino and El-Hakim, 2006) is an important tool nowadays for realistic 3D modeling and other applications in close range photogrammetry (CRP) and computer vision (CV). One crucial step within IBM is the image network planning and subsequent image orientation, or bundle adjustment. The following paper is concerned with those tasks. Today, different software is available for performing theimage orientation task automatically, either commercial like Photomodeler (PhotoModeler, 2009) and Photoscan (Photoscan,2011) or open-source like Bundler (Snavely, 2010), MICMAC (Pierrot-Deseilligny, 2012) and VSfM (Wu, 2012). However, there is no guarantee about the correct results and often the reliability is very low (Remondino et al., 2012) especially in the case of large datasets, wide baseline configurations, illumination changes, or when repetitive pattern and homogenous texture areas exist.

Image orientation can be achieved more reliably when certain information is available like initial camera exterior and interior orientation parameters, image overlapping information and information about the object geometry and structure.

Accordingly, the first demand of detecting image points and matching them with other correspondences in other images represents the most challenging tasks in the whole procedure (Remondino et al., 2012; Yang and Yuille, 1995) Matching is also a time dependent operation especially when the captured images $n$ are:

- of high resolution (HR), which is the case nowadays even with consumer compact cameras or smartphones.

- arranged in sparse block where the computation cost according to Barazzetti et al. (2010) is $O\left(n^{2}\right)$ with a combination of images $\left(\frac{n^{2}-n}{2}\right)$.

The paper will address those issues by a consequent exploitation of the knowledge about scene geometry and approximate camera positions. In Alsadik et al. (2013) an approach for a fully automatic camera network planning tool is described. Starting from a video capture of the object of interest, such as a building or a statue, and using established shape-from-motion techniques, a simple 3D model is created. Based on this model, 
the optimal camera locations for high resolution images are derived, where optimal refers to (i) retrieving a maximum point accuracy and (ii) keep the number of images at a minimum to reduce computation time. In a subsequent step, the camera operator is guided to those optimal places and asked to take the high resolution images (cf. section 2.1 below).

The paper continues along those research lines. Since the approximate orientations of high resolution images and the scene geometry are known, that knowledge is exploited for the task of correspondences matching and final image orientation, including bundle adjustment. Especially the fact that for each image its matching mates are known independently from actual matching techniques is an advantage over other approaches, such as presented in (Snavely et al., 2008). In those methods the so-called matching tree is computed from a brute-force matching (all images against all), because no pre-information about image locations is available. Those techniques are not only expensive in terms of computation time but also vulnerable to problems of mismatches, e.g. resulting from repetitive patterns or symmetries in building architecture (Kosecka and Zhang, 2010), as shown in Fig.1.

Image matching is followed by a bundle adjustment to orient the images automatically and to finally have a sparse 3D model. We use the commercial software Photomodeler Scanner 2010 (PhotoModeler, 2009) for implementing the bundle adjustment since it reports all the accuracy indices (quality and RMSE) which are necessary for the evaluation purposes. However, open source software might be used later in the bundle adjustment task like SBA (Lourakis and Argyros, 2004) and APERO (Pierrot-Deseilligny, 2012).



Figure 1. Repetitive texture patterns affecting the matching and leading to wrong correspondences.

\section{METHOD}

The research will investigate a robust methodology for the extraction of image correspondences following three successive steps:

Designing the camera network and image capture.

Computing the spanning tree of matching.

Guiding the multi windows matching by exploiting the rough object model.

\subsection{Camera network design and image capture}

Designing the imaging network (Fraser, 1996; Mason, 1995) is still a challenging task in photogrammetry due to many factors related to the nature and complexity of the study object, the used camera, accessibility and visibility. In Alsadik et al. (2013) an approach for optimal network design for cultural heritage objects (like buildings and statues) is presented. The method is based on creating a rough point cloud representing the object geometry from a video stream, employing a camera tracking or SfM techniques (Nister, 2001; Pollefeys et al., 2004). This point cloud is the base for designing a dense camera network that is filtered and optimized according to the coverage and accuracy requirement as shown in Fig. 2. The design will guarantee at least three covering cameras per point beside a total estimated error that fits the $3 \mathrm{D}$ modeling requirement. Therefore, with the assumption of using a pre-calibrated camera, the image interior and exterior orientation will be initially known.

A guiding procedure is followed on the basis of creating synthetic images. These synthetic images are important to guide the camera operator to capture the pre-designed image poses computed in the designing step. For each synthetic image an equivalent $3 \mathrm{D}$ point cloud is created. Therefore every pixel in the synthetic image will have the color information (RGB) and an assigned position information (XYZ), retrieved from the initial point cloud.

SIFT (Lowe, 2004) keypoint matching is implemented to measure whether the high resolution images are correctly captured. Finally, a space resection is performed for every acquired image in order to check the shift with respect to the designed orientation and to decide whether to proceed or to capture again the same image. After the guiding setup is finished, a complete set of HR images with their initial exterior orientation is available. Further information is available in (Alsadik et al., 2012, 2013).



Figure 2. Camera network design (Alsadik et al., 2012)

\subsection{Guided matching for correspondences extraction}

The guided image matching method will be presented in the following sections. Firstly, the advantages in developing the spanning tree of matching is discussed and secondly, the usage of the scene geometry and the initial camera orientations is presented.

2.2.1 Spanning tree of matching: The spanning tree of matching in our approach can be extracted by projecting back the object points that are used in the planning steps. This helps to decide their visibility status within the captured images by using:

The pre-designed exterior orientation parameters.

The surface points and normal directions.

The interior camera parameters.

Therefore, the decision of matching two images is decided by testing the existence of shared points that is visible in both images. Fig. 3 illustrates the spanning tree for the guided matching of 106 images around a building. The very dense tree of the large set of images indicates the necessary huge amount of data to be handled and processed. Snavely et al. (2008) presented their skeletal procedure for the matching tree in the case of a large, unordered and highly redundant sampled photo collection. However, this problem is already solved in our approach since, as mentioned before, the definite relation between the image pairs in the designing stage is known. 

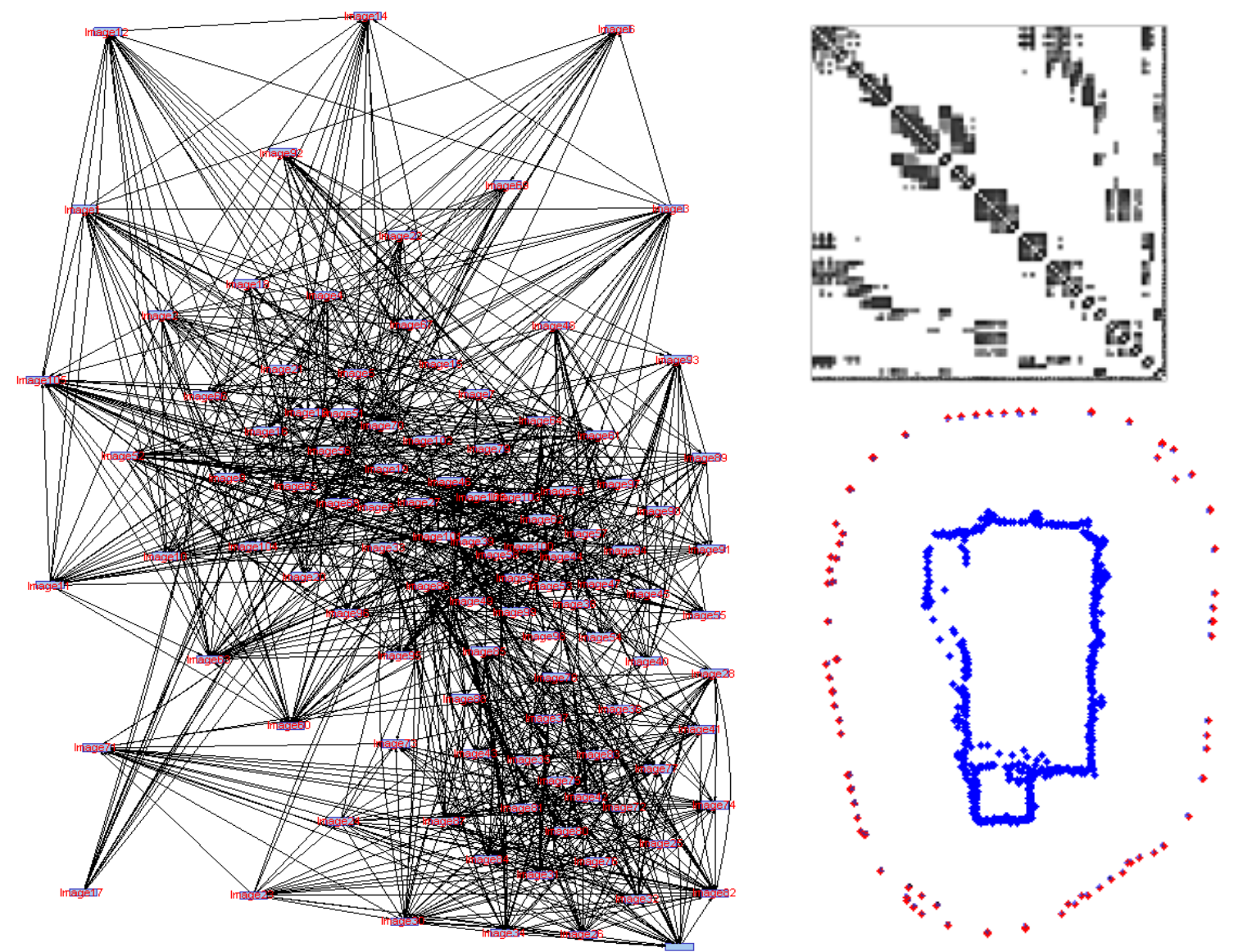

Figure 3. The spanning tree and the matching matrix of a pre-planned network of 106 images

2.2.2 Guided image matching by exploiting the object model: Once the spanning tree is defined, the correspondences matching is only done in stereo pairs which are connected within the tree. For the actual tie point matching in a stereo pair, methods which exploit the pre-knowledge of camera orientation and scene geometry are used, like the epipolar resampling approach of stereo images (Cho et al., 1992) or utilizing directly the initial 3D model. In this paper the guided matching is based on the second technique. This approach should strengthen the matching by providing a very good approximation for the corresponding points in the search images. In addition possible problems due to symmetries occurring at opposite façades will be avoided (e.g. Fig. 1).

The rough 3D scene geometry was already employed in the guiding of the camera operator: for each planned camera position a synthetic image was composed, also carrying the XYZ scene information per pixel. Those images are now being re-used. For each real image first a $2 \mathrm{D}$ affine transformation is estimated, because the real captured image does not fit exactly to the planned (synthetic image). The parameters for the transformation are computed using SIFT keypoints in both images (the synthetic and real image), which gets resampled to a similar resolution as the synthetic one. After the transformation the real image gets assigned the scene geometry as well.

For each stereo pair, first SIFT keypoints are extracted in one of the resampled real images. Since the XYZ position of those keypoints is known as well as the approximate exterior orientation of the stereo mate, each keypoint location can be projected into the stereo mate. The actual point matching is then done in the full resolution images, but restricted to windows defined around the estimated locations as shown in Fig. 4. The window size is a user-defined value since it depends on many factors like the resolution of the images, the initial image orientation, and the density of the tie points. As an alternative to SIFT we can use the SURF operator (Bay et al., 2008) and the normalized cross correlation (NCC) method.

In the next step outliers are filtered through a RANSAC procedure, using a F-Matrix estimation. Although we work currently with calibrated cameras, thus the more restrictive essential matrix E could be employed, some flexibility is kept, in order to be able to work also with un-calibrated cameras. For a more accurate localization of the tie points, the Least Square Matching (LSM) method (Gruen, 1985) is used to refine the localization of the matched points into sub-pixel accuracy (Barazzetti et al., 2010). Accurate matching will certainly improve the image orientation results and the $3 \mathrm{D}$ modeling task at the end.

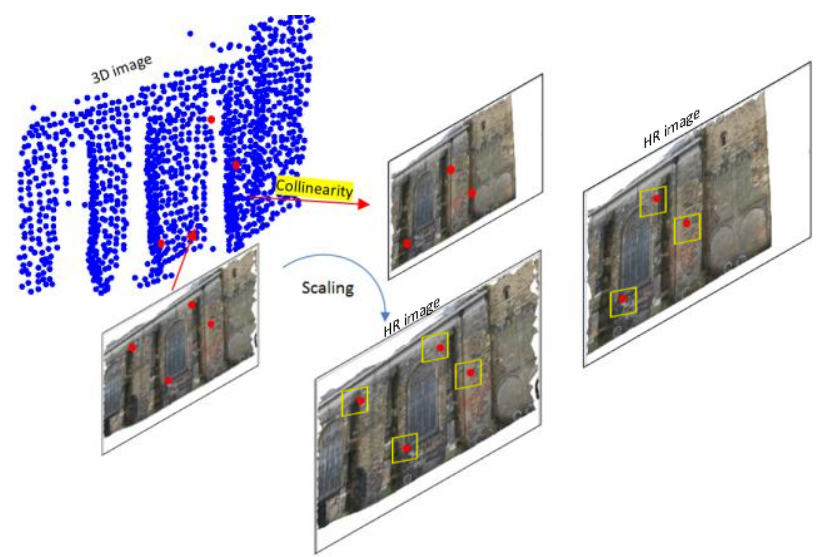

Figure 4. Keypoints estimation in the high resolution images 
Figure 5 illustrates the methodology of the whole guidedmatching technique.

The area of matching between the stereo images will be constrained to only the object itself. This is due to the method which is based on the previously derived rough $3 \mathrm{D}$ model of the object. This will limit the search space between the stereo images, avoid the mismatching points resulted from the dense texture in the background, and reduce the processing time. However, this might be a disadvantage if we need to implement the self-calibration due to the possible poor distribution of the points in some cases when the object spans a small portion of the images.

\section{Methodology for entire workflow}

A) Network planning and HR image capture

- Design the imaging network

- Create synthetic images and match later with real images

- Space resection for the orientation and validation of the real images

- Create 3D point cloud for each image during the synthesizing step

B) Guided matching exploiting approximate 3D structure and image orientation

- Compute the spanning tree of matching

- Compute affine transf. between the master synthetic and resized real image to model the slight shift

- SIFT keypoints detection of the master (left) real image

- Interpolate their XYZ object coordinates

- Use collinearity to estimate the homologous xy-image coordinates in the right image

Transform the xy-image points into the full resolution images

- Define a search window for each estimated point

- SIFT descriptor matching (or SURF, or NCC) in HR images for each window in the stereopairs

- Blunder detection and removal by RANSAC

- LSM for refinement

- Prepare and run bundle adjustment



Figure 5. The guided - Matching methodology by exploiting the 3D model

\section{EXPERIMENT}

To investigate the efficiency of our new method for guided correspondences matching, a pre-designed block of $30 \mathrm{HR}$ images of a monument is used, as shown in Fig. 6. The images are taken with a calibrated Canon 500D camera (15Mpx resolution) coupled with a $18 \mathrm{~mm}$ focal length.

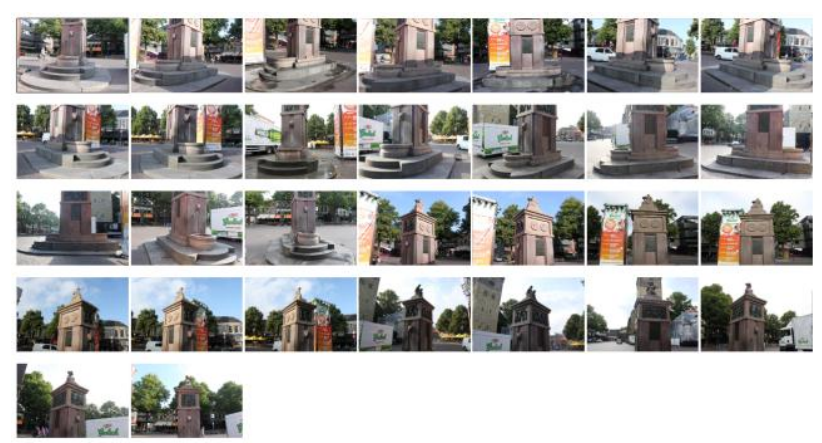

Figure 6. The dataset of 30 images of a monument (Alsadik et al., 2013).

\section{RESULTS}

The results will first present a comparison between the full pairwise matching tree (typical of SfM tools) and the guiding matching presented in this paper. This is done by using the SIFT GPU implemented in the VSfM tool. The computed matching matrices are illustrated in Fig. 7a, b. The computation of the spanning tree in the guided matching is done by using the initial exterior orientation of the design as described in section 2.1 .

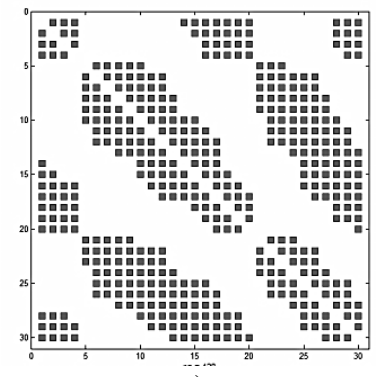

a)

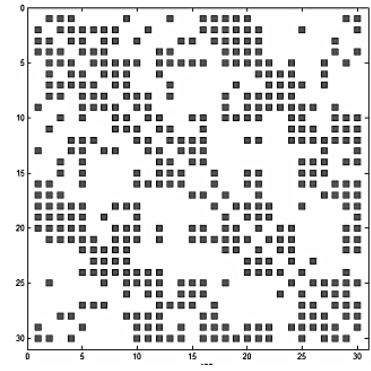

b)
Figure 7. The matching matrices: guided matching matrix according to the presented approach (a) and full pairwise matching matrix (b), typical of SfM approaches.

Fig. 8 shows a typical full pairwise mismatching result between images 4 and 13 of the dataset (despite the use of RANSAC for outlier rejection) obtained with VSfM. This is expected to be avoided in the guided matching procedure.

To elaborate more about the advantage of the implemented guided matching, the images were resampled into four different sizes. The full pairwise matching of VSfM and the developed method are then compared in terms of processing time. The results are presented in Fig. 9 and Table 1 showing the clear improvement of the proposed method. 


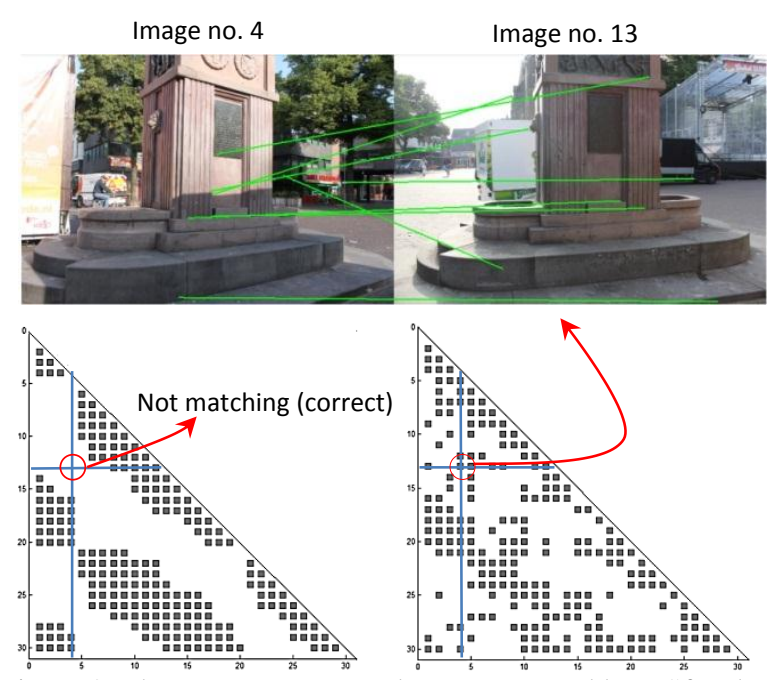

Figure 8 . The wrong correspondences extracted by VSfM due to symmetrical or repetitive texture and pattern.



Figure 9. The histogram shows the processing time for the full pairwise and the developed spanning tree.

\begin{tabular}{ccc}
\cline { 2 - 3 } & \multicolumn{2}{c}{ Processing time [seconds] } \\
\hline $\begin{array}{c}\text { Image width resolution } \\
\text { [pixels] }\end{array}$ & $\begin{array}{c}\text { Full pairwise } \\
\text { approach }\end{array}$ & $\begin{array}{c}\text { Spanning tree } \\
\text { approach }\end{array}$ \\
\hline 640 & 82 & 48 \\
\hline 1600 & 134 & 71 \\
\hline 1920 & 303 & 152 \\
\hline 2240 & 440 & 209 \\
\hline
\end{tabular}

Table 1. The processing time for the full pairwise and the precomputed spanning tree matching.

Moreover, the methodology of the guided matching is investigated by selecting three stereopairs of the monument dataset (Fig.6). The pairs are chosen in order to have wide and short image baseline configuration as well as moving objects in the background, as shown in Fig. 10.

The guided matching was implemented exploiting the rough model as previously described, initiating the SIFT or SURF keypoints in the low resolution images. The SIFT operator extracts, on the master image $(640 * 480)$, a total of 725 keypoints. Among these, 109 points located in the area of interest defined by the $3 \mathrm{D}$ model remain. The test shows the power and efficiency of the approach to find the approximate corresponding points.

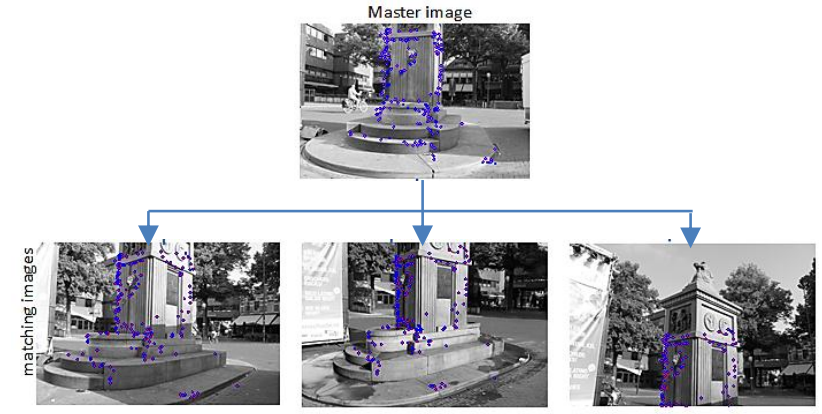

Figure 10. The four images and the matching approximation results of the guided approach

The keypoints are transformed into the full size of the images and define search windows for the final SIFT matching. Fig. 11 illustrates a sample of the estimated SIFT keypoints after scaling and the windows $(90 * 90$ pixel) matching results and the final matching points after outlier removal.

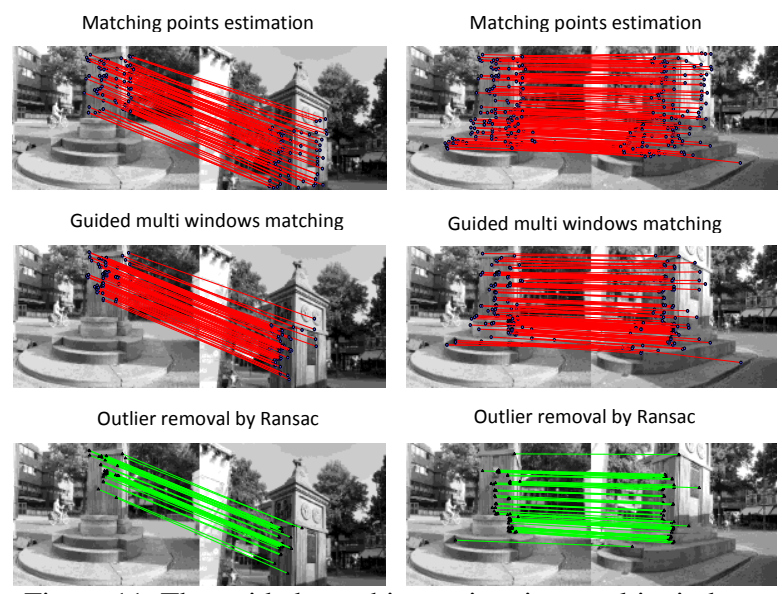

Figure 11. The guided matching estimation, multi windows matching and the results after the outliers removal.

The previous results show the performance of the approach for pairwise matching. Finally the approach is evaluated through passing the entire matching results and the approximate image orientation parameters to a bundle block adjustment algorithm, in our case the Photomodeler software. The results are illustrated in Fig. 12 and Table 2. Several interesting observations can be made. Only three images (2,3 and 4) are oriented in the commercial (PhotoModeler) and the open source (VSfM) software while the first image couldn't be oriented. In the guided matching approach based on SIFT, SURF, and NCC 256, 144 and 103 points are detected respectively which all belong to the study object and nothing is detected in the background. This is an advantage in the sense of avoiding mismatching due to repetitive pattern and moving objects.

It must be noted that the average point marking residual in the guiding matching as illustrated in Table 2 is larger than the residual in the commercial software. This is probably due to the fact that the distortion due to the wide baseline between the first and the other images affected the SIFT or SURF descriptor matching efficiency. Moreover, SIFT showed to be slightly more efficient than SURF in terms of precision results as shown in Table 2. 




a)

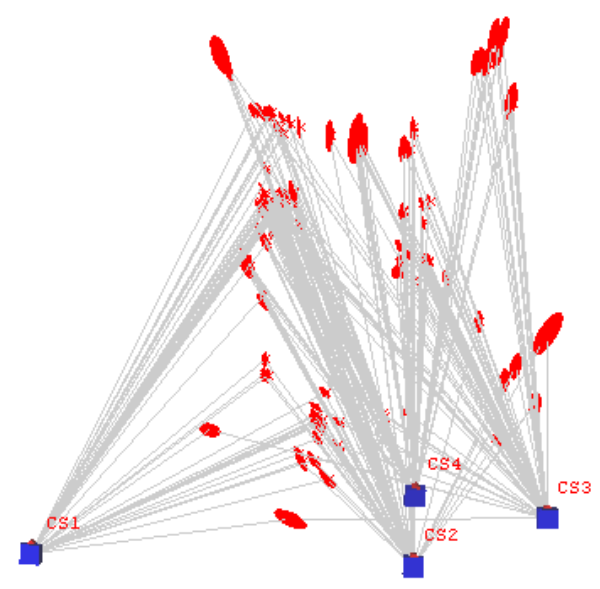

b)

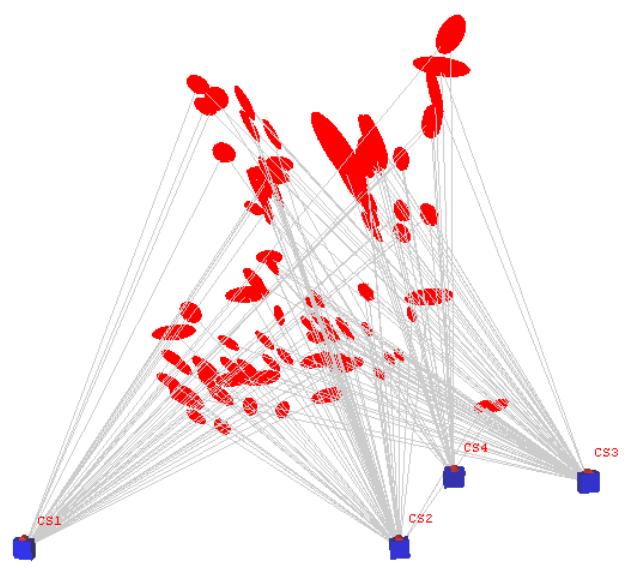

c)

Figure.12. The bundle adjustment results with exaggerated error ellipsoids: a) SIFT guided matching; b) SURF guided matching; c) NCC guided matching.

\begin{tabular}{|c|c|c|c|c|c|}
\hline & \multicolumn{3}{|c|}{ Guided matching } & \multirow[t]{2}{*}{ Photomodeler (smart match) } & \multirow[t]{2}{*}{ VSfM } \\
\hline & SIFT & SURF & $\mathrm{NCC}$ & & \\
\hline No. of oriented images & 4 & 4 & 4 & 3 & 3 \\
\hline No. of tie points & 256 & 144 & 103 & 287 & 304 \\
\hline Avg. point marking residuals [pixels] & 0.92 & 0.69 & 2.07 & 0.81 & - \\
\hline Avg. point precisions [RMSE] & $0.012 \mathrm{~m}$ & $0.013 \mathrm{~m}$ & $0.030 \mathrm{~m}$ & 0.143 pixel & 0.258 pixel \\
\hline Avg. point Angles [degrees] & 22 & 16 & 21 & 6 & - \\
\hline
\end{tabular}

Table 2. Comparison of automated image orientation in different approaches

\section{DISCUSSION AND CONCLUSIONS}

The paper presented a new method to extract image correspondences using a guided method. It combines two complementary image modeling techniques:

(i) a video-based $\mathrm{SfM}$ which is not sensitive to repetitive patterns and symmetries, because geometric feature tracking techniques can be used. Video-based SfM however does not lead to the best precision because of low resolution and short baseline image geometry.

(ii) wide baseline images for the final processing, leading to much better image ray intersection geometry and accuracy.

The inherent disadvantage of standard projects where no scene geometry can be used, namely the bad effects of repetitive patterns and object symmetries on image matching, do not influence our method.

The presented approach starts with the automated camera network design and a subsequent guided image capture. The image capture and validation is the final design step before the guided image matching is started. A dataset of 30 images $(4752 * 3168$ pixels) of a monument was used to show the efficiency of the method. The use of the pre-computed spanning tree proves its efficiency when compared with the full pairwise matching of common SfM methods (e.g. VSfM). In comparison with a full matching tree, the advantage of our pre-planned design is to avoid mismatching results as shown Fig. 8 and to reduce computation time for the processing of high-resolution images. 
The results obtained with the guided matching showed that the exploitation of the rough scene model and approximate image orientation provides a successful matching (see example in Fig.10) while state-of-the-art SfM techniques skipped one of the images.

The final accuracy of both approaches is somewhat comparable, but because different images are involved in the final solution a more detailed check needs to be done in the future.

As an additional future work, the epipolar resampling technique will be further investigated. One idea is to use it after the approach presented here, where the 3D geometry is exploited: the relative orientation of images is known very well and the epipolar resampling method can be used to find more tie points outside the object of interest. Secondly, the LSM refinement effect on the bundle adjustment will be evaluated too.

\section{REFERENCES}

Alsadik, B., Gerke, M., Vosselman, G., 2012. Optimal Camera Network Design for 3D Modeling of Cultural Heritage. ISPRS Annals of the Photogrammetry, Remote Sensing and Spatial Information Sciences, Volume I-3, pp.7-12. XXII ISPRS Congress, Melbourne, Australia.

Alsadik, B., Gerke, M., Vosselman, G., 2013. Automated camera network design for 3D modeling of cultural heritage objects. In: Journal of Cultural Heritage. Journal of Cultural heritage, In Press.

Barazzetti, L., Scaioni, M., Remondino, F., 2010. Orientation and 3D modelling from markerless terrestrial images: combining accuracy with automation. The Photogrammetric Record, 25(132), pp.356-381.

Bay, H., Ess, A., Tuytelaars, T., Van Gool, L., 2008. SpeededUp Robust Features (SURF). Computer Vision and Image Understanding, 110(3), pp.346-359.

Cho, W., Schenk, T., Madani, M., 1992. Resampling Digital Imagety to Epipolar Geometry, in: The Ohio State University, C., Ohio 43210-1247 (Ed.), Research Report No. 418.

Fraser, C.S., 1996. Network design, in: Atkinson, Close-range Photogrammetry and Machine Vision. Whittles Publishing UK pp. 256-282.

Gruen, A.W., 1985. Adaptive Least Squares Correlation: a Powerful Image Matching Technique. South African Journal of Photogrammetry, Remote Sensing and Cartography, 14(3), pp.176-189.

Kosecka, J., Zhang, W., 2010. Extraction, matching, and pose recovery based on dominant rectangular structures. Computer Vision and Image Understanding, 100, pp.274-293.

Lourakis, M.I.A., Argyros, A.A. (2004). "The Design and Implementation of a Generic Sparse Bundle Adjustment Software Package Based on the Levenberg - Marquardt Algorithm." from http://www.ics.forth.gr/ lourakis/sba/.

Lowe, D.G., 2004. Distinctive Image Features from ScaleInvariant Keypoints. Int. J. Comput. Vision, 60(2), pp.91-110.
Mason, S., 1995. Expert system- Based Design of Close-Range Photogrammetric Networks. ISPRS Journal of Photogrammetry and Remote Sensing, 50(5), pp.13-24.

Nister, D., 2001. Automatic Dense Reconstruction from Uncalibrated Video Sequence. Stockholm University.

PhotoModeler, 2009. PhotoModeler Quick Start Guide. www.photomodeler.com.

Photoscan, A. (2011). "AgiSoft StereoScan." from http://www.agisoft.ru/.

Pierrot-Deseilligny, M. (2012). "MicMac, software for automatic matching in the geographical context." from http://www.micmac.ign.fr/index.php?id=6.

Pollefeys, M., Gool, L.V., Vergauwen, M., Verbiest, F., Cornelis, K., Tops, J., Koch, R., 2004. Visual Modeling with a Hand-Held Camera. Int. J. Comput. Vision, 59(3), pp.207-232.

Remondino, F., El-Hakim, S., 2006. Image-based 3D modelling: A review. The Photogrammetric Record, 21(115), pp.269-291.

Remondino, F., Pizzo, S., Kersten, T., Troisi, S., 2012. LowCost and Open-Source Solutions for Automated Image Orientation - A Critical Overview, in: Ioannides, M., Fritsch, D., Leissner, J., Davies, R., Remondino, F., Caffo, R. (Eds.), Progress in Cultural Heritage Preservation. Springer Berlin Heidelberg, pp. 40-54.

Snavely, N. (2010). "Bundler: Structure from Motion (SfM) for Unordered Image Collections." from http://phototour.cs.washington.edu/bundler

Snavely, N., Seitz, S.M., Szeliski, R., 2008. Skeletal graphs for efficient structure from motion. In Proc. Computer Vision and Pattern Recognition (CVPR).

Wu, C. (2012). "VisualSFM : A Visual Structure from Motion System." from http://homes.cs.washington.edu/ ccwu/vsfm/.

Yang, Y., Yuille, A.L., 1995. Multilevel enhancement and detection of stereo disparity surfaces. Artificial Intelligence, 78, pp.121-145. 